October 25, 2018

\title{
Properties of equations of the continuous Toda type
}

\author{
${ }^{*}$ E. Alfinito, ${ }^{\dagger}$ G. Profilo, ${ }^{*}$ G. Soliani \\ * Dipartimento di Fisica dell'Università, 73100 Lecce, Italy, \\ and Istituto Nazionale di Fisica Nucleare, Sezione di Lecce, Italy. \\ † Dipartimento di Matematica dell'Università, 73100 Lecce, Italy.
}

\begin{abstract}
We study a modified version of an equation of the continuous Toda type in $1+1$ dimensions. This equation contains a friction-like term which can be switched off by annihilating a free parameter $\epsilon$. We apply the prolongation method, the symmetry and the approximate symmetry approach. This strategy allows us to get insight into both the equations for $\epsilon=0$ and $\epsilon \neq 0$, whose properties arising in the above frameworks are mutually compared. For $\epsilon=0$, the related prolongation equations are solved by means of certain series expansions which lead to an infinite- dimensional Lie algebra. Furthermore, using a realization of the Lie algebra of the Euclidean group $E_{2}$, a connection is shown between the continuous Toda equation and a linear wave equation which resembles a special case of a three-dimensional wave equation that occurs in a generalized Gibbons-Hawking ansatz. Nontrivial solutions to the wave equation expressed in terms of Bessel functions are determined.

For $\epsilon \neq 0$, we obtain a finite-dimensional Lie algebra with four elements. A matrix representation of this algebra yields solutions of the modified continuous Toda equation associated with a reduced form of a perturbative Liouville equation. This result coincides with that achieved in the context of the approximate symmetry approach. Example of exact solutions are also provided. In particular, the inverse of the exponential-integral function turns out to be defined by the reduced differential equation coming from a linear combination of the time and space translations. Finally, a Lie algebra characterizing the approximate symmetries is discussed.
\end{abstract}




\section{INTRODUCTION}

We investigate the equation

$$
u_{t t}+\epsilon u_{t}=\left(e^{u}\right)_{x x}
$$

where $u=u(x, t)$, subscripts denote partial derivatives and $\epsilon$ is a constant. For $\epsilon=0$, Eq.(11) is a continuous Toda system in $1+1$ dimensions (or, equivalently, a two-dimensional version of the so-called heavenly equation: self-dual Einstein spaces with a rotational Killing vector 2 ). The latter arises in many branches of physics, running from the theory of Hamiltonian systems, to the topological field theory. 3 In the case in which $\epsilon \neq 0$, the term $\epsilon u_{t}$ mimics a friction-like behaviour. Eq.(11), for $\epsilon \neq 0$ has been handled, in part, in Ref.[3] by means of an approximate group analysis.

We look for an algebraic characterization of Eq. (11) in both the cases $\epsilon=0$ and $\epsilon \neq 0$. In doing so, we resort to the prolongation method 6 and the symmetry approach. to these techniques to a minimum. Our main results are the following. For $\epsilon=0$, Eq. (11) can be written as a set of (prolongation) differential equations which can be solved in terms of power series expansions whose coefficients (in the variable $z=e^{u}$ ) depend on the pseudopotential components and obey a presumably infinite-dimensional Lie algebra.

A remarkable fact is that this algebra can be closed "step by step", in the sense that a finite-dimensional Lie algebra emerges in correspondence of each polynomial in $z$ coming from the truncation of the series. The use of a given representation of any closed Lie algebra allows us to find a linear problem associated with the equation under consideration. Furthermore, we show that the prolongation differential equations related to Eq. (1) $(\epsilon=0)$ afford a class of solutions connected with the Lie algebra of the Euclidean group $E_{2}$ in the plane. This enables us to map Eq. (1) into the linear wave equation

$$
y_{t t}-e^{u} y_{x x}=0,
$$

where $y=y(x, t)$ is a pseudopotential variable. We point out that Eq.(2) is equivalent to a $1+1$ dimensional version of Eq.(2) of ref.[1] in which a generalized Gibbons-Hawking ansatz11 pertinent to a quantum theory of gravity is

considered. Starting from simple solutions of Eq. (1), examples of nontrivial solutions of Eq. (2) are displayed.

In the case $\epsilon \neq 0$, the prolongation algebra for Eq. (1) turns out to be finitedimensional. This algebra is exploited to determine a class of special solutions via a reduced form of a Liouville-type equation. The latter coincides with that arising in the context of the symmetry approach. This feature suggests the existence of a possible link between the prolongation method and the symmetry approach, which deserves further studies. Finally, a self-similar solution in terms of the exponential-integral function is obtained. 
The paper is organized as follows. In Sec. II, the prolongation equations derived for Eq. (11) are studied. In Sections III and IV, the cases $\epsilon=0$ and $\epsilon \neq 0$ are considered, respectively. Section V, deals with the symmetry and the approximate symmetry approach applied to Eq. (1D). Precisely, the generators of the Lie point symmetries and approximate symmetries are found. The latter can be characterized by a finite dimensional Lie algebra which admits a realization in terms of boson annihilation and creation operators. In Sec. VI, the main results are summarized and some comments are reported. Finally, Appendices $\mathrm{A}$ and $\mathrm{B}$ contain details of calculation, while Appendix $\mathrm{C}$ is devoted to a brief introduction to the approximate symmetry method.

\section{THE PROLONGATION EQUATIONS}

Let us consider the prolongation system for Eq. (11):

$$
y_{x}^{i}=F^{i}\left(u, u_{t} ; y^{j}\right), \quad y_{t}^{i}=G^{i}\left(u, u_{x} ; y^{j}\right),
$$

where $i, j=1,2, \ldots, N$ ( $N$ arbitrary), and the set of variables $\left\{y^{i}\right\}$ is the pseudopotential. 6 The compatibility condition for Eq. (3) yields

$$
F^{i}=L^{i} u_{t}+M^{i}, \quad G^{i}=L^{i} e^{u} u_{x}+P^{i},
$$

where $M^{i}=M^{i}\left(u ; y^{j}\right), P^{i}=P^{i}\left(u ; y^{j}\right)$, and $L^{i}=L^{i}\left(y^{j}\right)$ are defined by

$$
\begin{gathered}
M_{u}^{i}+[P, L]^{i}=\epsilon L^{i}, \\
e^{u}[L, M]^{i}=P_{u}^{i}, \\
{[M, P]^{i}=0,}
\end{gathered}
$$

where $[P, L]^{i}=P^{k} \frac{\partial L^{i}}{\partial y_{k}}-L^{k} \frac{\partial P^{i}}{\partial y_{k}}$, and so on.

For brevity, hereafter we shall omit the index $i$. Now, we look for a solution of Eq. (5 8 ) of the form

$$
M=\sum_{k=0}^{\infty} a_{k}(y) z^{k}, \quad P=\sum_{k=0}^{\infty} b_{k}(y) z^{k}
$$

where $z=e^{u}$ and $y$ stands for the set of components $\left\{y^{j}\right\}(j=1,2, \ldots, N)$.

By inserting the expansions (8) into Eqs.(阿团), we obtain the following constraints between the coefficients $a_{k}(y)$ and $b_{k}(y)$ :

$$
\begin{gathered}
{\left[b_{0}, L\right]=\epsilon L,} \\
{\left[L, b_{k}\right]=k a_{k},} \\
{\left[L, a_{k-1}\right]=k b_{k},}
\end{gathered}
$$




$$
\begin{gathered}
{\left[a_{0}, b_{0}\right]=0,} \\
{\left[a_{0}, b_{1}\right]+\left[a_{1}, b_{0}\right]=0,} \\
{\left[a_{0}, b_{2}\right]+\left[a_{1}, b_{1}\right]+\left[a_{2}, b_{0}\right]=0,} \\
\ldots \ldots \ldots \ldots \ldots \ldots \ldots \ldots \ldots \ldots \ldots \ldots \ldots \ldots \\
\sum_{k=1}^{N}\left[a_{k-1}, b_{N-k}\right]=0,
\end{gathered}
$$

where $k=1,2, \ldots N$ ( $N$ arbitrary).

In order to scrutinize the commutation relations (9-15), two cases have to be distinguished, i.e. $\epsilon=0$ and $\epsilon \neq 0$.

\section{THE CASE $\epsilon=0$}

Let us assume $\epsilon=0$ in Eqs. (9)-(15). Then, the systematic application of the Jacobi identity to the commutation relations (9)-(15) produces an arbitrary number of finite- dimensional Lie algebras with $2 \mathrm{~N}+1$ generators $(\mathrm{N}=1,2, \ldots)$, i.e. $a_{0}, a_{1}, \ldots, a_{N}, b_{0}, b_{1} \ldots, b_{N}$, and $L$ ( $N$ arbitrary).

Although at present a rigorous proof is not given, this statement can be checked heuristically "step by step" in the sense explained below.

To this aim, let us take (first step) $a_{j}=0, b_{j}=0$, for $j=1,2 \ldots$. Thus, from Eqs. (9)- (15) we get the abelian Lie algebra

$$
\left[a_{0}, b_{0}\right]=0, \quad\left[a_{0}, L\right]=0, \quad\left[b_{0}, L\right]=0 .
$$

Any realization of this algebra corresponds to a solution of the type $M=$ $a_{0}(y), P=b_{0}(y)$, to Eqs.(河)-(7) (with $\epsilon=0$ ). In this case, the prolongation system (3, 画) becomes

$$
y_{x}=L u_{t}+a_{0}, \quad y_{t}=L e^{u} u_{x}+b_{0} .
$$

Of course, the compatibility condition for the system (17) is fulfilled if (16) holds.

Now, let us choose (second step) $a_{j}=0, b_{j}=0$, for $j=2,3, \ldots$ From (9)-(15) we have (see Appendix A)

$$
\begin{gathered}
{\left[a_{0}, a_{1}\right]=0, \quad\left[a_{0}, b_{0}\right]=0, \quad\left[a_{0}, b_{1}\right]=0, \quad\left[a_{0}, L\right]=-b_{1}} \\
{\left[a_{1}, b_{0}\right]=0, \quad\left[a_{1}, b_{1}\right]=0, \quad\left[a_{1}, L\right]=0, \quad\left[b_{0}, b_{1}\right]=0} \\
{\left[b_{0}, L\right]=0, \quad\left[b_{1}, L\right]=-a_{1} .}
\end{gathered}
$$

Equations (18) represent a non-abelian Lie algebra defined by the five elements $a_{0}, a_{1}, b_{0}, b_{1}$ and $L$. Any realization of the algebra (18) corresponds to a solution of the type

$$
M(u, y)=a_{0}(y)+a_{1}(y) e^{u},
$$




$$
P(u, y)=b_{0}(y)+b_{1}(y) e^{u},
$$

to Eqs.(5)-(河). The prolongation equations (3)-(何) related to (19)-(20) are

$$
y_{x}=L u_{t}+a_{0}+a_{1} e^{u}, \quad y_{t}=L e^{u} u_{x}+b_{0}+b_{1} e^{u} .
$$

The compatibility condition for this system is verified by the algebra (18).

Furthermore, as it is shown in Appendix A, under the hypothesis $a_{j}=$ $0, \quad b_{j}=0$, for $j=3,4, \ldots$ (third step), Eqs.(9)-(14) give rise to the prolongation Lie algebra defined by the seven elements $a_{0}, a_{1}, a_{2}, b_{0}, b_{1}, b_{2}$, and $L$ :

$$
\begin{gathered}
{\left[a_{0}, a_{1}\right]=0, \quad\left[a_{0}, a_{2}\right]=0, \quad\left[a_{0}, b_{0}\right]=0, \quad\left[a_{0}, b_{1}\right]=0} \\
{\left[a_{0}, b_{2}\right]=0, \quad\left[a_{0}, L\right]=-b_{1}, \quad\left[a_{1}, a_{2}\right]=0, \quad\left[a_{1}, b_{0}\right]=0} \\
{\left[a_{1}, b_{1}\right]=0, \quad\left[a_{1}, b_{2}\right]=0, \quad\left[a_{1}, L\right]=-2 b_{2}, \quad\left[a_{2}, b_{0}\right]=0} \\
{\left[a_{2}, b_{1}\right]=0, \quad\left[a_{2}, b_{2}\right]=0, \quad\left[a_{2}, L\right]=0, \quad\left[b_{0}, b_{1}\right]=0,} \\
{\left[b_{0}, b_{2}\right]=0, \quad\left[b_{0}, L\right]=0, \quad\left[b_{1}, b_{2}\right]=0,} \\
{\left[b_{1}, L\right]=a_{1}, \quad\left[b_{2}, L\right]=-2 a_{2} .}
\end{gathered}
$$

The prolongation equations (3)-(14) can be written as

$$
y_{x}=L u_{t}+a_{0}+a_{1} e^{u}+a_{2} e^{2 u}, \quad y_{t}=L e^{u} u_{x}+b_{0}+b_{1} e^{u}+b_{2} e^{2 u},
$$

whose compatibility condition is ensured by (22).

The next step consists in finding a closed Lie algebra defined by the nine generators $a_{0}, a_{1}, a_{2}, a_{3}, b_{0}, b_{1}, b_{2}, b_{3}$, and $L$ (see Appendix A). Since the character of the commutation relations (A1)-(A 12) is basically of the recursive type, we expect that the procedure of building up finite-dimensional Lie algebras starting from (9)-(15) $(\epsilon=0)$ works out for any step. In other words we have the following possible scenario: Equation (1),$(\epsilon=0)$ admits the prolongation Lie algebra defined by the commutation relations (see Appendix A):

$$
\begin{gathered}
{\left[L, a_{l-1}\right]=l b_{l},\left[L, a_{N}\right]=0,\left[L, b_{k}\right]=k a_{k},} \\
{\left[a_{j}, a_{k}\right]=0,\left[a_{j}, b_{k}\right]=0,\left[b_{j}, b_{k}\right]=0,}
\end{gathered}
$$

for $j, k,=0,1,2, \ldots, N$, and $l=1,2, \ldots, N$. Since $N$ is an arbitrary positive integer, Eqs.(24) represent an infinite-dimensional Lie algebra.

Another interesting result concerning the case $\epsilon=0$, is expressed by the following 
Proposition 1 Let $u$ be a solution of the equation

$$
u_{t t}=\left(e^{u}\right)_{x x} .
$$

Then, the function $y_{2}=y_{2}(x, t)$ defined by

$$
\begin{gathered}
y_{2 x}=-i \mathcal{C}_{0}(\xi) \cosh y_{1}, \\
y_{2 t}=-\frac{1}{2} \xi \mathcal{C}_{1}(\xi) \sinh y_{1}, \\
y_{1 x}=u_{t}, \quad y_{1 t}=e^{u} u_{x},
\end{gathered}
$$

satisfies the wave equation

$$
y_{2 t t}-e^{u} y_{2 x x}=0,
$$

where $\mathcal{C}_{0}(\xi)$, with $\xi=2 i e^{\frac{u}{2}}$, fulfills the differential equation of the Bessel type

$$
\frac{d^{2} \mathcal{C}_{0}}{d \xi^{2}}+\frac{1}{\xi} \frac{d \mathcal{C}_{0}}{d \xi}+\mathcal{C}_{0}=0
$$

and

$$
\mathcal{C}_{1}=i \frac{d \mathcal{C}_{0}}{d \xi}
$$

To prove this Proposition, let us search a solution to Eqs.(5.7) $(\epsilon=0)$ of the form

$$
M(u, y)=m(u) g(y), \quad P(u, y)=p(u) h(y) .
$$

Substitution from (32) into Eqs. (局, yields

$$
m_{u}=p, \quad m e^{u}=p_{u},
$$

and

$$
[L, h]=g, \quad[L, g]=h, \quad[g, h]=0 .
$$

Equations (33) provide

$$
m_{u u}-m e^{u}=0, \quad p_{u u}-p_{u}=e^{u} p,
$$

which give

$$
m=\mathcal{C}_{0}(\xi), \quad p=-\frac{i}{2} \xi \mathcal{C}_{1}(\xi),
$$

respectively, where $\xi=2 i e^{\frac{u}{2}}$, and $\mathcal{C}$ denotes a function of the Bessel type $J, Y, H^{(1)}, H^{(2)}$, or any linear combination of them.

We notice that by setting $L=i X_{1}, h=X_{2}$ and $g=i X_{3}$, Eqs.(34) become:

$$
\left[X_{1}, X_{2}\right]=X_{3}, \quad\left[X_{1}, X_{3}\right]=X_{2}, \quad\left[X_{2}, X_{3}\right]=0
$$


i.e. the Lie algebra associated with the Euclidean group, $E_{2}$, in the plane.

A realization of Eqs. (37) in terms of a two-component pseudopotential $y=\left(y_{1}, y_{2}\right)$ is

$$
X_{1}=-i \partial_{y_{1}}, \quad X_{2}=-i \sinh y_{1} \partial_{y_{2}}, \quad X_{3}=-\cosh y_{1} \partial_{y_{2}} .
$$

Then, Eqs.(3) can be written as [see (41) and (3)]:

$$
\begin{gathered}
y_{1 x}=u_{t}, \quad y_{1 t}=e^{u} u_{x}, \\
y_{2 x}=-i m \cosh y_{1}, \quad y_{2 t}=-i p \sinh y_{1} .
\end{gathered}
$$

Thus, Eqs. (26-27) are nothing but (40) with $m$ and $p$ replaced by the quantities (36). Furthermore, Eq. (29) arises straightforwardly form Eqs. (40) with the help of Eqs. (33). Finally, Eqs. (30) and (31) are a direct consequence of Eqs. (4) and (9)-(15).

We observe also that by combining together Eqs.(29) and (25) we can express Eqs.25 by means of the pseudopotential component $y_{2}$, namely,

$$
\partial_{t}^{2} \ln \frac{y_{2 t t}}{y_{2 x x}}=\partial_{x}^{2} \frac{y_{2 t t}}{y_{2 x x}} .
$$

Below we shall display a few examples of nontrivial solutions of the wave equation (29), starting from some simple solutions of Eq.(25).

To this aim, let us consider the simple solution $u=t$ to Eq. 225). Then, by choosing $\mathcal{C}_{0}(\xi)=i J_{0}(\xi)$, so that $\mathcal{C}_{1}(\xi)=-\frac{d J_{0}(\xi)}{d \xi}=J_{1}(\xi)$ [see Eq. (36)], where $J_{0}$ and $J_{1}$ are Bessel functions of the first kind, from Eq. (40) we obtain

$$
\begin{gathered}
y_{2 x}=J_{0}(\xi) \cosh x, \\
y_{2 t}=-\frac{\xi}{2} J_{1}(\xi) \sinh x,
\end{gathered}
$$

where $\xi=2 i e^{\frac{t}{2}}\left[y_{1 x}=1, \quad y_{1 t}=0\right.$; see Eq. (39)] $]$.

Equations (42)-(43) can be easily integrated to give

$$
y_{2}(x, t)=J_{0}\left(2 i e^{\frac{t}{2}}\right) \sinh x .
$$

Hence, the pseudopotential variable (44) represents a particular solution to the wave equation

$$
y_{2 t t}-e^{t} y_{2 x x}=0 .
$$

Another application of the previous Proposition concerns the equation

$$
y_{2 t t}-x y_{2 x x}=0,
$$


which corresponds to $u=\ln x$ [a special solution to Eq.(25)]. Using the same procedure as before, after easy calculations we find

$$
y_{2}=\frac{1}{2} \xi \frac{d J_{0}(\xi)}{d \xi} \cosh t,
$$

where $\xi=2 i x^{\frac{1}{2}}$.

At this point it is instructive to show that the explicit form of the pseudopotential can be used to solve certain linear second order ordinary differential equations with variable coefficients. In fact, by way of example, let us put

$$
y_{2}=f(x) g(t)
$$

into Eq. (46). Then, Eq. (46) entails

$$
\begin{gathered}
g_{t t}-g=0, \\
f_{x x}-\frac{1}{x} f=0 .
\end{gathered}
$$

Now, since $g=\cosh t$ is a particular integral of Eq.(48), from (47) and (48) we get

$$
f(x)=-i x^{\frac{1}{2}} J_{1}\left(2 i x^{\frac{1}{2}}\right) .
$$

\section{THE CASE $\epsilon \neq 0$}

For $\epsilon \neq 0$, the prolongation algebra of Eq.(1) is a Lie algebra $\mathcal{L}$ closed at the beginning (see Appendix A). This reads

$$
\begin{gathered}
{\left[a_{0}, b_{0}\right]=\left[a_{0}, b_{1}\right]=\left[L, b_{1}\right]=0,} \\
{\left[b_{0}, b_{1}\right]=\epsilon b_{1},} \\
{\left[b_{0}, L\right]=\epsilon L,} \\
{\left[L, a_{0}\right]=b_{1} .}
\end{gathered}
$$

A matrix representation of $\mathcal{L}$ is

$$
\begin{array}{cl}
a_{0}=\left(\begin{array}{ccc}
0 & -1 & 0 \\
0 & 0 & 0 \\
0 & 0 & 0
\end{array}\right), & b_{0}=\left(\begin{array}{ccc}
\epsilon & 0 & 0 \\
0 & \epsilon & 0 \\
0 & 0 & 0
\end{array}\right), \\
b_{1}=\left(\begin{array}{lll}
0 & 0 & 1 \\
0 & 0 & 0 \\
0 & 0 & 0
\end{array}\right), \quad L=\left(\begin{array}{lll}
0 & 0 & 0 \\
0 & 0 & 1 \\
0 & 0 & 0
\end{array}\right) .
\end{array}
$$


In view of (56), Eqs.(3) take the form

$$
\begin{aligned}
& \left(\begin{array}{l}
y_{1} \\
y_{2} \\
y_{3}
\end{array}\right)_{x}=\left(\begin{array}{ccc}
0 & 0 & 0 \\
-1 & 0 & 0 \\
0 & u_{t} & 0
\end{array}\right)\left(\begin{array}{l}
y_{1} \\
y_{2} \\
y_{3}
\end{array}\right), \\
& \left(\begin{array}{l}
y_{1} \\
y_{2} \\
y_{3}
\end{array}\right)_{t}=\left(\begin{array}{ccc}
\epsilon & 0 & 0 \\
0 & \epsilon & 0 \\
e^{u} & e^{u} u_{x} & 0
\end{array}\right)\left(\begin{array}{l}
y_{1} \\
y_{2} \\
y_{3}
\end{array}\right),
\end{aligned}
$$

from which $y_{1}=\lambda_{1} e^{\epsilon t}, y_{2}=\zeta e^{\epsilon t}$ and

$$
\begin{gathered}
y_{3 \zeta}=-\frac{1}{\lambda_{1}} e^{\epsilon t} u_{t} \zeta, \\
y_{3 t}=-\lambda_{1} e^{\epsilon t} \zeta^{2} \frac{\partial}{\partial \zeta} \frac{e^{u}}{\zeta},
\end{gathered}
$$

where $\zeta=-\lambda_{1} x+\lambda_{2}$, and $\lambda_{1}, \lambda_{2}$ are constants of integration. Here $y_{3}$ plays the role of a potential variable.

We remark that Eqs.(59)-(60) can be exploited to determine explicit solutions of Eq.(11) $(\epsilon \neq 0)$. In doing so, let us seek, for instance, solutions of the type $u_{t}=\gamma(t)$. After some manipulations, Eqs. (59) and (60) provide

$$
u=\ln \left[\frac{1}{2 \lambda_{1}^{2}}\left(\gamma_{t}+\epsilon \gamma\right) \zeta^{2}+\alpha \zeta+e^{-\epsilon t} \Gamma_{t}\right],
$$

where $\gamma=\gamma(t), \alpha=\alpha(t)=e^{\beta}$ and $\Gamma=\Gamma(t)$ are such that

$$
\begin{gathered}
\gamma=\frac{\alpha_{t}}{\alpha}, \\
\beta_{t t}+\epsilon \beta_{t}=c_{1} e^{\beta}, \\
\Gamma_{t}=c_{2} e^{\int d t(\gamma+\epsilon)},
\end{gathered}
$$

with $c_{1}, c_{2}$ constants of integration.

It is noteworthy that Eq. (63), which is a modified version of the reduced Liouville equation, has the same form of that obtained in the framework of the Lie group approach $\mathbf{t}$ via a certain symmetry variable (see Sec.V).

In the simple case $c_{1}=0$, Eq.(63) is easily solved. Then we find the exact solution

$$
u=k_{1} e^{-\epsilon t}+\ln \left(e^{\frac{k_{2}}{\epsilon}} \zeta+k_{0}\right)
$$

to Eq.(1)), where $k_{0}, k_{1}$ and $k_{2}$ are arbitrary constants. Otherwise (when $c_{1} \neq 0$ and $\epsilon$ is a small parameter), Eq.(53) can be analyzed by using some perturbative technique. 


\section{THE SYMMETRY APPROACH}

\section{A. Symmetry generators}

As is well known, in the study of a system of partial differential equations one can use symmetry groups to find special solutions (which are invariants under some subgroups of the complete symmetry group) by solving reduced systems of differential equations involving fewer independent variables than the original system. However, the standard technique has to be modified if small perturbations are present in the equations under consideration. In this context, the authors of Ref. [2] devised a method based on the concepts of approximate group of transformations and approximate symmetries. For the reader's convenience, in Appendix $\mathrm{C}$ we shall recall the main aspects of this method.

In order to obtain the approximate symmetries admitted by Eq.(值) $(\epsilon \neq 0)$, let us take in Eqs. (C20) and (C21):

$$
\begin{gathered}
F_{0}=u_{t t}-\left(e^{u}\right)_{x x}, \quad F_{1}=u_{t t} \\
z=\left(t, x, u, u_{x}, u_{t t}, u_{t x}, u_{x x}\right), \\
X=\left(\xi_{0}^{1}+\epsilon \xi_{1}^{1}\right) \partial_{t}+\left(\xi_{0}^{2}+\epsilon \xi_{1}^{2}\right) \partial_{x}+\left(\xi_{0}^{3}+\epsilon \xi_{1}^{3}\right) \partial_{u}, \\
\xi^{j}=\left(\xi_{o}^{3}\right)^{j}+\epsilon\left(\xi_{1}^{3}\right)^{j}, \quad j=4, \ldots, 8,
\end{gathered}
$$

where

$$
\begin{aligned}
& \left(\xi_{\alpha}^{3}\right)^{J}=D_{J}\left(\xi_{\alpha}^{3}-\sum_{i=1}^{2} u_{i}^{\alpha}\right)+\sum_{i=1}^{2} \xi_{\alpha}^{i} u_{J, i}^{\alpha}, \\
& u_{i}^{\alpha}=\frac{\partial u^{\alpha}}{\partial x^{i}}, \quad u_{J, i}^{\alpha}=\frac{\partial u_{J}^{\alpha}}{\partial x^{i}}, \quad \alpha=0,1,
\end{aligned}
$$

$D_{J}$ denotes the total derivative with respect to $J=t, x, t t, t x, x x$, respectively, and $x^{1} \equiv t, x^{2} \equiv x$.

By expliciting $(\overline{\mathrm{C} 20})$ and $(\overline{\mathrm{C} 21})$ and keeping in mind (56)-(58), equating the coefficients of the independent variables $u_{0 t}, u_{0 t} u_{0 x}, \ldots$ to zero, we arrive at the following set of constraints:

$$
\begin{gathered}
\xi_{j}^{3}+2 \xi_{j t}^{1}-2 \xi_{j x}^{2}=0, \quad \xi_{j x x}^{2}-2 \xi_{j x}^{2}=0, \\
2 \xi_{j t u}^{3}-\xi_{j t t}^{1}=0, \quad \xi_{j t t}^{3}-e^{u_{0}} \xi_{j x x}^{3}=0, \quad(j=0,1),
\end{gathered}
$$

and

$$
2 \xi_{j t u}^{3}-\xi_{j t t}^{1}+c_{j}=0,
$$

where $\xi_{j}^{1}=\xi_{j}^{1}(t), \xi_{j}^{2}=\xi_{j}^{2}(x), \xi_{j}^{3}=\xi_{j}^{3}(x, t)$, and $c_{j}$ is a constant of integration. 
By solving Eqs. (69) and (70), the expression (66) takes the form

$$
\begin{aligned}
X & =\left[c_{1} t+c_{2}+\epsilon\left(\frac{c_{1}}{2} t^{2}+k_{1} t+k_{2}\right)\right] \partial_{t} \\
& +\left[\left(c_{1}+c_{3}\right) x+c_{4}+\epsilon\left(\frac{k_{3}}{2}+k_{1}\right) x+\epsilon k_{4}\right] \partial_{x} \\
& +\left[2 c_{3}+\epsilon\left(-2 c_{1} t+k_{3}\right)\right] \partial_{u},
\end{aligned}
$$

with $c_{1}, c_{2}, \ldots$ and $k_{1}, k_{2}, \ldots$ arbitrary constants.

From the quantity (71) we have the operators

$$
\begin{aligned}
& X_{1}=X_{1}^{0}+\epsilon\left(\frac{t^{2}}{2} \partial_{t}-2 t \partial_{u}\right), \quad X_{2} \equiv X_{2}^{0}=\partial_{t} \\
& X_{3} \equiv X_{3}^{0}=\partial_{x} \quad X_{4} \equiv X_{4}^{0}=x \partial_{x}+2 \partial_{u}
\end{aligned}
$$

where

$$
X_{1}^{0}=t \partial_{t}+x \partial_{x}
$$

and

$$
\begin{aligned}
& X_{5}=\epsilon\left(t \partial_{t}+x \partial_{x}\right), \quad X_{6}=\epsilon \partial_{t}, \\
& X_{7}=\frac{\epsilon}{2}\left(x \partial_{x}+2 \partial_{u}\right), \quad X_{8}=\epsilon \partial_{u} .
\end{aligned}
$$

The operators $X_{1}^{0}, X_{2}^{0}, X_{3}^{0}, X_{4}^{0}$ are the (exact) symmetry generators relative to Eq.(11) for $\epsilon=0$, while $X_{1}, X_{2}, X_{3}, X_{4}$ are the approximate generators of Eq.(11) for $\epsilon \neq 0$. The exact symmetry generators of Eq. (11) ) for $\epsilon \neq 0$ are $X_{1}^{0}, X_{2}^{0}$ and $X_{3}^{0}$. The operators (74) are inessential, in the sense that $\epsilon$ is a constant factor.

\section{B. Algebraic properties}

The operators (72), (73) obey the commutation relations

$$
\begin{gathered}
{\left[X_{2}, X_{1}\right]=X_{2}+\epsilon\left(t \partial_{t}-2 \partial_{u}\right),} \\
{\left[X_{2}, X_{4}\right]=0, \quad\left[X_{2}, X_{3}\right]=0 \quad\left[X_{1}, X_{4}\right]=0,} \\
{\left[X_{1}, X_{3}\right]=-X_{3}, \quad\left[X_{3}, X_{4}\right]=X_{3} .}
\end{gathered}
$$

We notice that the commutation rules (75)-(77) do not define a (finite) Lie algebra. However, they can be used to build up a realization of a finite-dimensional Lie algebra by introducing the "auxiliary" operators

$$
Z=t \partial_{t}-2 \partial_{u}, \quad Y=\frac{t^{2}}{2} \partial_{t}-2 t \partial_{u} .
$$


In doing so, it turns out that $Y, Z, X_{j}(j=1, \ldots, 4)$ satisfy the commutation relations

$$
\begin{gathered}
{\left[X_{2}, X_{1}\right]=X_{2}+\epsilon Z, \quad\left[X_{3}, X_{1}\right]=X_{3}, \quad\left[X_{1}, X_{4}\right]=0} \\
{\left[X_{1}, Y\right]=Y, \quad\left[X_{1}, Z\right]=-\epsilon Y} \\
{\left[X_{2}, Y\right]=Z, \quad\left[X_{2}, Y\right]=X_{2}, \quad[Z, Y]=Y} \\
{\left[X_{2}, X_{3}\right]=\quad\left[X_{2}, X_{4}\right]=\left[X_{3}, X_{4}\right]=\left[X_{3}, Y\right]=0} \\
{\left[X_{4}, Y\right]=\left[X_{3}, Z\right]=\left[X_{4}, Z\right]=0 .}
\end{gathered}
$$

For brevity, by the symbols $Y, Z, X_{j}(j=1, \ldots, 4)$ we shall indicate both the abstract elements and the corresponding realization (72)-(73) and (75)-(77) of the finite-dimensional Lie algebra (79)-( 82).

Now, let us focus our attention on the subalgebra (81). This is isomorphic to the $\operatorname{sl}(2, R)$ algebra given by

$$
\left[Z^{\prime}, T\right]=2 S, \quad[T, S]=2 Z^{\prime}, \quad\left[S, Z^{\prime}\right]=-2 T,
$$

where

$$
T=\sqrt{2}\left(Y+X_{2}\right), \quad S=\sqrt{2}\left(Y-X_{2}\right), \quad Z^{\prime}=2 Z .
$$

Furthermore, the following Propositions hold:

Proposition 2 The Casimir operator

$$
\begin{aligned}
C & =T^{2}-S^{2}-Z^{\prime 2} \\
& \equiv 4\left[2 X_{2} Y-Z(Z+1)\right]
\end{aligned}
$$

of the Lie algebra (83), commutes with all the generators $Y, Z, X_{j}(j=1, \ldots, 4)$ of the Lie algebra (79)-(89).

The proof is straightforward.

Proposition 3 The algebra (79)-89) admits a realization in terms of boson annihilation and creation operators.

This can be shown by putting

$$
a_{1}^{\dagger}=t, \quad a_{2}^{\dagger}=u, a_{3}^{\dagger}=x, a_{1}=\partial_{t}, a_{2}=\partial_{u}, \quad a_{3}=\partial_{x},
$$

to yield

$$
\left[a_{j}, a_{k}^{\dagger}\right]=\delta_{j k}, \quad\left[a_{j}, a_{k}\right]=0 \quad(j, k=1,2,3)
$$




$$
\begin{aligned}
& Y=\frac{1}{2} a_{1}^{\dagger}{ }_{1}^{2} a_{1}-2 a_{1}^{\dagger} a_{2}, \quad Z=a_{1}^{\dagger} a_{1}-2 a_{2}, \\
& X_{1}=a_{1}^{\dagger} a_{1}+a_{3}^{\dagger} a_{3}+\epsilon\left(\frac{1}{2} a_{1}^{\dagger} a_{1}^{2}-2 a_{1}^{\dagger} a_{2}\right), \\
& X_{2}=a_{1}, \quad X_{3}=a_{3}, \quad X_{4}=a_{3}^{\dagger} a_{3}+2 a_{2},
\end{aligned}
$$

and

$$
C=-8 a_{2}\left(a_{2}+1\right) .
$$

We would like to finish this Subsection with a remark on the "auxiliary" operators (78). These are essential for establishing the closed algebra (79)-(82). Notwithstanding, their meaning is not completely clear. For instance it should be important to ascertain whether finite-dimensional Lie algebra analogous to (79)-(82) can be constructed in relation to other case studies. At present, we are able to affirm only that the operators (78), $Z$ and $Y$ can be interpreted, respectively, as symmetry variables of the equations

$$
\begin{gathered}
u_{x x}+\epsilon u_{x}=-\left(e^{-u}\right)_{t t}, \\
u_{x x}+\epsilon u_{x}=-2\left(e^{-\frac{u}{2}}\right)_{t t},
\end{gathered}
$$

which arise, formally from Eq. (11) via the transformations $t \rightarrow x, u \rightarrow-u$, and $t \rightarrow x, u \rightarrow-\frac{u}{2}$. From $Z$ we obtain the invariant $\eta(x) \equiv e^{q(x)}=t e^{\frac{u}{2}}$, that once replaced into Eq.(91) yields the reduced equation of the modified Liouville type

$$
q_{x x}+\epsilon q_{x}=-e^{-2 q} .
$$

In a similar way, from (92) we find

$$
r_{x x}+\epsilon r_{x}=-4 e^{-\frac{r}{2}}
$$

where the invariant $r(x)=u+4 \ln t$ associated with $Y$ has been used.

\section{Explicit solutions}

To see how the symmetry approach works out, we shall deal with two significant examples. First, let us write down the group transformations related to the generator $X_{4} \equiv X_{4}^{0}$ [see $(72)$, which is present in both the cases $\epsilon=0$ and $\epsilon \neq 0$. We have

$$
x^{\prime}=e^{\lambda} x, t^{\prime}=t, u^{\prime}=u+2 \lambda,
$$

where $\lambda$ is the group parameter.

From (95) we deduce the invariant

$$
\rho=x^{\prime}=e^{-\frac{u^{\prime}}{2}}=x=e^{-\frac{u}{2}} .
$$


Then, making use of (96), Eq. (1) provides the reduced equation

$$
\rho_{t}^{2}-\rho \rho_{t t}-\epsilon \rho \rho_{t}=1,
$$

which is transformed in the (ordinary) equation of the modified Liouville type

$$
w_{t t}+\epsilon w_{t}=-\epsilon^{-2 w}
$$

through the change of variable $\rho=e^{w}$. We notice that by setting $w=-\beta$, Eq. (98) coincides with Eq. (63) for $c_{1}=2$.

This property may be interpreted as a hint of a possible connection between the prolongation algebra and the symmetry generators coming from the group analysis. This important aspect pertinent to the algebraic theory of nonlinear field equations is a challenging subject for further investigations.

For $\epsilon=0$, Eq. (98) is solved by

$$
w=\ln \frac{\cos \sqrt{c}\left(t-t_{0}\right)}{\sqrt{c}},
$$

where $c$ and $t_{0}$ are positive constants.

Therefore, from (96) we obtain the exact solution

$$
u=2 \ln \frac{\sqrt{c} x}{\cos \sqrt{c}\left(t-t_{0}\right)}
$$

to Eq. (1) (with $\epsilon=0$ ).

We point out that the function (100) can be derived from Eqs. (59) and (60) as a special case. In fact by choosing $\epsilon=0$, Eqs.(57) and (58) yield

$$
y_{3 x}=\left(\lambda_{2}-\lambda_{1} x\right) u_{t}, \quad y_{3 t}=e^{u}\left[\lambda_{1}+\left(\lambda_{2}-\lambda_{1} x\right) u_{x}\right],
$$

from which

$$
e^{u}=\frac{1}{2} \gamma_{t} x^{2}
$$

where we have assumed $u_{t}=\gamma(t), \lambda_{2}=0$, and the functions of integration have been taken equal to zero. From Eq.(102) we get $u_{t}=\frac{\gamma_{t t}}{\gamma_{t}} \equiv \gamma$, i.e. $\gamma(t)=2 \sqrt{c} \tan \sqrt{c}\left(t-t_{0}\right)$. Consequently, Eq.(102) reproduces just the solution (100).

This result is not surprising, since the requirement $u_{t}=\gamma(t)$ means that we single out a class of solutions to Eq. $(1)(\epsilon=0)$ corresponding to the symmetry reduction based on the invariant $\rho(t)$ associated with the generator $X_{4}^{0}$. The relation between $\rho(t)$ and $\gamma(t)$ is $-2 \frac{\rho_{t}}{\rho}=\gamma$ [see (96)]. Finally, the algebra (52)(55) with $\epsilon=0$ coincides with that derived from (18) for $a_{1}=0$ (see Appendix A). 
Second, let us deal with the vector field obtained from the linear combination of the generators $X_{3}=X_{3}^{0}=\partial_{x}$ and $X_{2}=X_{2}^{0}=\partial_{t}$ :

$$
V=v \partial_{x}+\partial_{t}
$$

where $v$ is a constant.

The group transformations read $x^{\prime}=x+v \lambda, t^{\prime}=t+\lambda$, which furnish the invariant

$$
\sigma=x^{\prime}-v t^{\prime}=x-v t .
$$

This quantity can be exploited to find a self-similar solution to Eq.(1). In fact, inserting $u=u(\sigma)$ into Eq.(1) we get

$$
\left(v^{2}-e^{u}\right) u_{\sigma}=\epsilon v u+c_{0},
$$

where $c_{0}$ is a constant of integration.

By setting

$$
u=-\tau-\ln v
$$

and $c_{0}=-2 \epsilon v \ln v$, Eq. (105) provides

$$
\int_{0}^{\tau_{1}} \frac{1-e^{-\tau \prime}}{\tau^{\prime}} d \tau^{\prime}=\frac{\epsilon}{v}\left(\sigma-\sigma_{0}\right)
$$

$\left(\xi_{0}=\right.$ constant $)$.

The left-hand side of (107) is the exponential-integral functiont

$$
\operatorname{Ein}(\tau)=\mathrm{E}_{1}(\tau)+\ln \tau+\gamma,
$$

where $\mathrm{E}_{1}(\tau)=\int_{\tau}^{\infty} \frac{e^{-\tau^{\prime}}}{\tau^{\prime}} d \tau^{\prime}$ and $\gamma$ is the Euler constant. Hence, from (106), (107) and (108) we have the self-similar solution

$$
u=-\operatorname{Ein}^{-1}\left[\frac{\epsilon}{v}\left(\sigma-\sigma_{0}\right)\right]-\frac{c_{0}}{\epsilon v},
$$

where $\operatorname{Ein}^{-1}(\cdot)$ denotes the inverse function of (108). We notice that the reduced equation (105) can be considered as an ordinary differential equation which defines the special function $\operatorname{Ein}^{-1}(\cdot)$.

\section{CONCLUSIONS}

We have studied an modified version of a continuous Toda equation in $1+1$ dimensions.

We have applied jointly two procedures: the prolongation method and the symmetry approach, which are mostly based on the use of algebraic and group techniques. This strategy reveals efficacious both from a conceptual point of 
view and for practical purposes, e.g. for the determination of exact solutions of the equations under consideration.

For $\epsilon=0$, on the basis of heuristic arguments we have found that an infinitedimensional prolongation Lie algebra may be associated with Eq. (1). This algebra can be closed step by step in the sense explained in Sec.III. In correspondence of any representation of a given finite-dimensional Lie algebra in terms of pseudopotential variables, one obtains a linear problem for Eq.(1). Moreover, a link is established between Eq.(11) and the wave equation (37). This connection derives from a special realization of the Lie algebra of the Euclidean group $E_{2}$ related to a class of solutions of the prolongation equations (5)-(7) $(\epsilon=0)$. It is noteworthy that nontrivial solutions to Eq.(37) expressed in terms of particular Bessel functions are determined. We remark also that explicit forms of the pseudopotential can be used to solve certain second order ordinary differential equations with non constant coefficients (see, for example, Eq.(58)).

For the case $\epsilon \neq 0$, we have shown that the prolongation algebra is finitedimensional and is constituted by four elements. A matrix representation of this algebra (see Eq.(64)) allows us to write Eq.(11) in a potential form which leads to solutions associated with those admitted by a modified version of the reduced Liouville equation (71). This equation has the same form of that coming from the symmetry approach via the generator $X_{4} \equiv X_{4}^{0}=x \partial_{x}+2 \partial_{u}$. This property indicates the existence of a possible connection between the prolongation method and the symmetry approach. This is an important methodological aspect which deserves a wide investigation. Here we remark only that any approach to this problem should not ignore the contribution by Harrison and Estabrook 10 where the fundamental concepts of Cartan's theory of systems of partial differential equations are exploited for obtaining the generators of their invariance groups (isogroups). Another interesting result achieved in the framework of the symmetry approach is given by Eq.(109), which tells us that the inverse of the exponential-integral function turns out to be defined by the reduced differential equation (105) corresponding to the generator $V=v \partial_{x}+\partial_{t}$.

We shall conclude with a few comments.

First, we have not tackled the problem of the complete integrability of Eq.(1) (for $\epsilon=0$ ). Anyway, as it happens for other nonlinear partial differential equations of physical interest, the existence of an infinite-dimensional prolongation algebra is necessary for the integrability property. However, in this regard, we recall that completely integrable nonlinear field equations admit Kac-Moody prolongation algebras endowed with a loop structure. Therefore, a definitive answer on the complete integrability of Eq. (1) $(\epsilon=0)$ is strictly connected with a deep study of its associated algebra (24). The situation is different for $\epsilon \neq 0$, in which a finite-dimensional prolongation algebra is found at the beginning. This feature indicates that Eq.(1) for $\epsilon \neq 0$ is not completely integrable.

Second, in the context of the approximate symmetry approach, i.e. when $\epsilon$ is a small parameter, a realization of a finite-dimensional Lie algebra, i.e. (79)- 
(82), can be constructed by introducing the auxiliary operators (78). This realization can be expressed in terms of boson annihilation and creation operators. The algebra (79)-(82), in some sense, seems to characterize the approximate symmetry of Eq.(1), but its role is not yet clear. For instance, may algebras of this type arise in the study of the approximate symmetries of other perturbative systems? Finally, our results could be useful in the study of a three-dimensional extension of Eq.(1), using the same theoretical frameworks of this paper. Concerning this point it should be interesting to see, in analogy with what happens in our case, whether the equations of Proposition 1 of Ref. [1]:

$$
\begin{gathered}
u_{x x}+u_{y y}+\left(e^{u}\right)_{z z}=0, \\
w_{x x}+w_{y y}+\left(w e^{u}\right)_{z z}=0,
\end{gathered}
$$

share a property similar to that linking Eqs.(25) and (29), through a representation of a Lie algebra of an extended Euclidean group. 


\section{Appendix A. : CLOSED LIE ALGEBRAS FROM (9)-(15) WITH $\epsilon=0$}

Exploiting the Jacobi identity, from (10) and (11) we obtain

$$
\begin{gathered}
{\left[a_{k}, a_{k^{\prime}}\right]=-\frac{1}{k^{\prime}}\left\{\left[L,\left[b_{k^{\prime}}, a_{k}\right]\right]-(k+1)\left[b_{k^{\prime}}, b_{k+1}\right]\right\},} \\
{\left[b_{k^{\prime}}, a_{k}\right]=-\frac{1}{k}\left\{\left[L,\left[b_{k}, b_{k^{\prime}}\right]\right]-k^{\prime}\left[b_{k}, a_{k^{\prime}}\right]\right\},} \\
{\left[b_{k}, b_{k^{\prime}}\right]=-\frac{1}{k^{\prime}}\left\{\left[L,\left[a_{k^{\prime}-1}, b_{k}\right]\right]-k\left[a_{k^{\prime}-1}, a_{k}\right]\right\} .}
\end{gathered}
$$

Equations (A2) and $\mathrm{A} 3 \mathrm{~B}$ ) provide

$$
\begin{gathered}
{\left[b_{0}, a_{k}\right]=\frac{1}{k}\left[L,\left[b_{0}, b_{k}\right]\right],} \\
{\left[b_{0}, b_{k}\right]=-\frac{1}{k}\left[L,\left[a_{k-1}, b_{0}\right]\right],}
\end{gathered}
$$

which give

$$
\left[b_{0}, a_{k}\right]=\frac{1}{(k !)^{2}}\left[L,\left[L, \ldots,\left[L,\left[L,\left[b_{0}, a_{1}\right]\right] \ldots\right]\right.\right.
$$

and

$$
\left[b_{0}, b_{k}\right]=\frac{1}{k[(k-1) !]^{2}}\left[L,\left[L,\left[b_{0}, a_{1}\right]\right] \ldots\right]
$$

where the operator $L$ on the right hand side of (A6) and (A7) appears 2(k-1) and 2(k-1)-1 times, respectively.

Other useful relations are

$$
\begin{gathered}
{\left[a_{0}, a_{k}\right]=-\frac{1}{k}\left\{\left[L,\left[b_{k}, a_{0}\right]\right]-(k+1)\left[b_{k}, b_{1}\right]\right\},} \\
{\left[a_{0}, b_{k}\right]=\frac{1}{k}\left\{\left[L,\left[a_{0}, a_{k+1}\right]\right]+\left[a_{k-1}, b_{1}\right]\right\},} \\
{\left[a_{k}, b_{k}\right]=\frac{1}{k}\left\{\left[L,\left[a_{k}, a_{k-1}\right]\right]+(k+1)\left[a_{k-1}, b_{k+1}\right],\right\},}
\end{gathered}
$$

where

$$
\left[a_{k}, a_{k-1}\right]=\frac{1}{k}\left[L,\left[b_{k}, a_{k-1}\right]\right] .
$$

We have also

$$
\left[b_{k-1}, b_{k}\right]=\frac{1}{k}\left[L,\left[b_{k-1}, a_{k-1}\right]\right] .
$$

Now, let us suppose that $a_{j}=0$, for $\mathrm{j}=2,3, \ldots$. Then, from Eq. (9), (10) and (11) we obtain

$$
\left[b_{0}, L\right]=0,\left[L, a_{0}\right]=b_{1},\left[L, b_{1}\right]=a_{1} .
$$


Furthermore, from (A4) and (13):

$$
\left[b_{0}, a_{1}\right]=0,\left[a_{0}, b_{1}\right]=0,
$$

while

$$
\left[a_{0}, a_{1}\right]=0
$$

from $\mathrm{A} 8$. We have also

$$
\left[b_{0}, b_{1}\right]=0
$$

by commuting Eq. (12) with L. Finally, (A10) provides

$$
\left[a_{1}, b_{1}\right]=0
$$

since $b_{2}=0$. The previous commutation relations define the algebra (27).

Now, let us suppose that $a_{j}=0, b_{j}=0$, for $\mathrm{j}=3,4, \ldots$. Then, Eq. (7) yields

$$
\begin{gathered}
{\left[a_{0}, b_{0}\right]=0,} \\
{\left[a_{0}, b_{1}\right]+\left[a_{1}, b_{0}\right]=0,} \\
{\left[a_{0}, b_{2}\right]+\left[a_{1}, b_{1}\right]+\left[a_{2}, b_{0}\right]=0,} \\
{\left[a_{1}, b_{2}\right]+\left[a_{2}, b_{1}\right]=0,} \\
{\left[a_{2}, b_{2}\right]=0 .}
\end{gathered}
$$

By commuting (A18) with $L$ and using the Jacobi identity, with the help of (9) $(\epsilon=0)$ and 111 we find

$$
\left[b_{0}, b_{1}\right]=0 .
$$

Taking account of (A23), Eq. A4) gives

$$
\left[b_{0}, a_{1}\right]=0 .
$$

Hence, from (A19):

$$
\left[a_{0}, b_{1}\right]=0 .
$$

Furthermore, Eqs. A11), (A25) and A10) give

$$
\begin{aligned}
& {\left[a_{1}, a_{0}\right]=0,} \\
& {\left[a_{1}, b_{1}\right]=0 .}
\end{aligned}
$$

Since

$$
\left[a_{2}, b_{0}\right]=0
$$

(see (A6) and (A24)), from (A19) we deduce

$$
\left[a_{0}, b_{2}\right]=0 .
$$


On the other hand, from (A3) (see (A27)):

$$
\left[b_{1}, b_{2}\right]=0
$$

while $(\mathrm{A2})$ provides

$$
\left[b_{2}, a_{1}\right]=2\left[b_{1}, a_{2}\right] .
$$

Keeping in mind A31, from A21 we have

$$
\left[b_{1}, a_{2}\right]=\left[b_{2}, a_{1}\right]=0 .
$$

We get also

$$
\left[a_{2}, a_{1}\right]=0,
$$

from (A1) and (A2). Finally, (A8) gives (see $\mathrm{A} 29$ and (A30):

$$
\left[a_{0}, a_{2}\right]=0 .
$$

Thus, all the commutators among the elements $a_{j}, b_{j}(j=0,1,2)$ have been determined. The commutators of the type $\left[a_{j}, L\right]$ and $\left[b_{j}, L\right]$ are expressed by (10) and (11). Therefore, we have the seven- dimensional Lie algebra (31).

Another example of finite-dimensional Lie algebra emerging from the commutation relations (95)-(15), involves the nine generators $a_{j}, b_{j}$ and $L$ with $j=0,1,2,3$. This algebra can be obtained following essentially the same scheme adopted for the construction of the algebra (31).

In fact, by putting

$$
\begin{gathered}
M=a_{0}+a_{1} z+a_{2} z^{2}+a_{3} z^{3}, \\
P=b_{0}+b_{1} z+b_{2} z^{2}+b_{3} z^{3},
\end{gathered}
$$

Eq.(7) entails

$$
\begin{gathered}
{\left[a_{0}, b_{0}\right]=0,} \\
{\left[a_{0}, b_{1}\right]+\left[a_{1}, b_{0}\right]=0,} \\
{\left[a_{0}, b_{2}\right]+\left[a_{1}, b_{1}\right]+\left[a_{2}, b_{0}\right]=0,} \\
{\left[a_{0}, b_{3}\right]+\left[a_{1}, b_{2}\right]+\left[a_{2}, b_{1}\right]+\left[a_{3}, b_{0}\right]=0,} \\
{\left[a_{1}, b_{3}\right]+\left[a_{2}, b_{2}\right]+\left[a_{3}, b_{1}\right]=0,} \\
{\left[a_{2}, b_{3}\right]+\left[a_{3}, b_{2}\right]=0,} \\
{\left[a_{3}, b_{3}\right]=0 .}
\end{gathered}
$$

Equations $(\mathrm{A36})-(\mathrm{A} 38)$ have been already examined. We need to scrutinize only those relations which contain $a_{3}$ and $b_{3}$, i.e. Eqs. A39)-(A42).

Since

$$
\left[a_{3}, b_{0}\right]=0
$$


(see $(\mathrm{A6})$ and $(\mathrm{A} 24)$ ), Eq. (A39) becomes

$$
\left[a_{0}, b_{3}\right]+\left[a_{1}, b_{2}\right]+\left[a_{2}, b_{1}\right]=0 .
$$

Now, by commuting (

$$
\begin{aligned}
& 3\left[a_{0}, b_{3}\right]=\left[a_{2}, b_{1}\right], \\
& 2\left[b_{1}, a_{2}\right]=\left[b_{2}, a_{1}\right] .
\end{aligned}
$$

Combining together $(\mathrm{A} 44),(\mathrm{A} 45)$ and $(\mathrm{A} 46)$ we arrive at

$$
\begin{aligned}
& {\left[a_{0}, b_{3}\right]=0,} \\
& {\left[a_{2}, b_{1}\right]=0,} \\
& {\left[a_{1}, b_{2}\right]=0 .}
\end{aligned}
$$

From ( $\mathrm{A} 8)$ and $(\mathrm{A} 47)$ :

$$
\left[a_{0}, a_{3}\right]=\frac{1}{3}\left[b_{3}, b_{1}\right] .
$$

On the other hand, Eq. (A47) and A49) give

$$
\left[a_{1}, a_{2}\right]=0 .
$$

Thus, using (A3), (A48) and (A51):

$$
\left[b_{1}, b_{3}\right]=0 .
$$

Hence, from (A50):

$$
\left[a_{0}, a_{3}\right]=0 .
$$

At this stage, let us consider the relation (A10) for $k=2$. We have

$$
\left[b_{2}, a_{2}\right]=\frac{3}{2}\left[a_{1}, b_{3}\right] .
$$

Furthermore, from ( $\mathrm{A2}$ ) and ( $\mathrm{A} 52)$ :

$$
\left[b_{3}, a_{1}\right]=3\left[b_{1}, a_{3}\right] .
$$

Now, by elaborating the commutator $\left[L,\left[a_{0}, a_{3}\right]\right]$ by means of the Jacobi identity (see (A53)) and taking $b_{4}=0$, we get

$$
\left[a_{3}, b_{1}\right]=0 .
$$

Thus, (A55) implies

$$
\left[b_{3}, a_{1}\right]=0,
$$


so that (A54) yields

$$
\left[a_{2}, b_{2}\right]=0 .
$$

Inserting (A58) in (A12) for $k=3$, we have

$$
\left[b_{2}, b_{3}\right]=0 .
$$

Then, from (A1):

$$
\left[a_{1}, a_{3}\right]=0,
$$

via (A52) and (A59).

To conclude, Eq.(A52) provides

$$
3\left[b_{2}, a_{3}\right]=2\left[b_{3}, a_{2}\right]
$$

[see A59)]. Therefore, Eq. (A61) and (A41) furnish

$$
\begin{aligned}
& {\left[b_{3}, a_{2}\right]=0,} \\
& {\left[b_{2}, a_{3}\right]=0 .}
\end{aligned}
$$

We have also

$$
\left[a_{2}, a_{3}\right]=0,
$$

from (A1) and (A62). Therefore, by collecting all the results achieved in the case $a_{j}=0, b_{j}=0(j=4,5, \ldots)$, the finite-dimensional Lie algebra of elements $a_{0}, a_{1}, a_{2} a_{3}, b_{0}, b_{1}, b_{2}, b_{3}$ and $L$ turns out to be defined by the commutation relations:

$$
\begin{aligned}
{\left[a_{j}, a_{k}\right] } & =0, \quad\left[a_{j}, b_{k}\right]=0, \quad\left[b_{j}, b_{k}\right]=0, \\
{\left[L, a_{l-1}\right] } & =l b_{l}, \quad\left[L, a_{3}\right]=0,\left[L, b_{k}\right]=k a_{k},
\end{aligned}
$$

for $j, k=0,1,2,3$ and $l=1,2,3$. 


\section{Appendix B. : FINITE DIMENSIONAL PROLONGATION ALGEBRA $(\epsilon \neq 0)$}

Here we prove that the prolongation algebra associated with Eq. (1) with $\epsilon \neq 0$ is the finite-dimensional Lie algebra (52)-(55). In doing so, let us start from the commutation rule

$$
\left[L, b_{1}\right]=a_{1},
$$

coming from (10) for $k=1$.

Then, by exploiting the Jacobi identity we have

$$
\left[L,\left[b_{0}, b_{1}\right]\right]+\epsilon a_{1}=\left[b_{0}, a_{1}\right],
$$

with the help of (9). On the other hand, the relation (11) (with $k=1$ ) provides

$$
\left[b_{0}, b_{1}\right]=\epsilon b_{1},
$$

by elaborating the commutator $\left[b_{0},\left[L, a_{0}\right]\right]$ via the Jacobi identity. Now, substitution from (B3) into (B2) gives

$$
\left[b_{0}, a_{1}\right]=2 \epsilon a_{1} .
$$

Taking account of (B4), Eq.(13) yields

$$
\left[a_{0}, b_{1}\right]=2 \epsilon a_{1} .
$$

At this point, let us consider the commutator $\left[b_{1}\left[a_{0}, b_{0}\right]\right]$ (see $(121)$ ), By resorting again to the Jacobi identity and using (B3), (B4) and (B5) we obtain the constraint

$$
\epsilon^{2} a_{1}=0 \text {. }
$$

Let us suppose that $\epsilon \neq 0$. Hence, Eq. B6 implies $a_{1} \equiv 0$. Consequently, from Eqs. (10) and (11) we infer that $2 b_{2}=\left[L, a_{1}\right]=0, \quad 2 a_{2}=\left[L, b_{2}\right]=0$, $3 b_{3}=\left[L, a_{2}\right]=0,3 a_{3}=\left[L, b_{3}\right]=0$, and so on. Therefore, for $\epsilon \neq 0$ the quantities $a_{0}, a_{1}, b_{0}, b_{1}$, and $L$, define the closed Lie algebra $\mathcal{L}$ expressed by (52)-(55). 


\section{Appendix C. : THE APPROXIMATE LIE GROUP ANAL- YSIS}

Here we recall some basic concepts inherent to the approximate group analysis of differential equations derived by Baikov, Gazizov and Ibragimov (BGI) in 1988. The starting point of this approach is a Theorem (see below) that allows one to construct approximate symmetries which are stable for small perturbations of the differential equation under investigation.

To describe briefly the BGI method let us consider the one-parameter group of local point transformations:

$$
z^{\prime}=g(z, \epsilon, a)
$$

where $z=\left(z_{1}, \ldots, z_{N}\right)$ is the independent variable, and $a$ is the group parameter so that the value $a=0$ corresponds to the identity transformation $g(z, \epsilon, a)=z$,

$$
g(g(z, \epsilon, a), \epsilon, b)=g(z, \epsilon, a+b),
$$

and $\epsilon$ is a perturbative parameter. Then let us suppose that $f \approx g$, namely

$$
f(z, \epsilon, a)=g(z, \epsilon, a)+o\left(\epsilon^{p}\right)
$$

for some fixed values of $p \geq 0$.

The transformations

$$
z^{\prime} \approx f(z, \epsilon, a)
$$

form an approximate one-parameter group $f$

$$
\begin{gathered}
f(z, \epsilon, 0) \approx z, \\
f(f(z, \epsilon, a), \epsilon, b) \approx f(z, \epsilon, a+b),
\end{gathered}
$$

and the condition $f(z, \epsilon, a) \approx z$ for all $z$ implies that $a=0$.

The following Theorem holds:

Theorem 1 Let us assume that the transformations (C4) form an approximate group with the tangent vector field

$$
\left.\xi(z, \epsilon) \approx \frac{\partial f(z, \epsilon, a)}{\partial a}\right|_{a=0}
$$

Then, the function $f(z, \epsilon, a)$ satisfies

$$
\frac{\partial f(z, \epsilon, a),}{\partial a} \approx \xi(f(z, \epsilon, a)) .
$$

Conversely, for any (smooth) function $\xi(z, \epsilon)$ the solution (C4) of the approximate Cauchy problem

$$
\frac{d z^{\prime}}{d a} \approx \xi\left(z^{\prime}, \epsilon\right),
$$




$$
\left.z^{\prime}\right|_{a=0} \approx z,
$$

determines an approximate one-parameter group with parameter $a$.

The previous Theorem is called the approximate Lie theorem, while Eq. (C9) is called the approximate Lie equation.

Resorting to the approximate Lie Theorem, we can construct the approximate group of transformations generated by a given infinitesimal operator. To see how the method works out, let us deal with the case $p=1$. Then we look for the approximate group of transformations

$$
z^{\prime} \approx f_{0}(z, a)+\epsilon f_{1}(z, a),
$$

determined by the infinitesimal operator

$$
X=\left(\xi_{0}(z)+\epsilon \xi_{1}(z)\right) \frac{\partial}{\partial z} .
$$

The related approximate Lie equation

$$
\frac{d}{d a}\left(f_{0}+\epsilon f_{1}\right) \approx \xi_{0}\left(f_{0}(z)+\epsilon f_{1}\right)+\epsilon \xi_{1}\left(f_{0}(z)+\epsilon f_{1}\right)
$$

becomes the system

$$
\frac{d f_{0}}{d a} \approx \xi_{0}\left(f_{0}\right), \quad \frac{d f_{1}}{d a} \approx \xi_{0}^{\prime}\left(f_{0}\right) f_{1}+\xi_{1}\left(f_{0}\right),
$$

where $\xi_{0}^{\prime}$ is the derivative of $\xi_{0}$.

The initial condition $\left.z^{\prime}\right|_{a=0} \approx z$ provides $\left.f_{0}\right|_{a=0} \approx z$ and $\left.f_{1}\right|_{a=0} \approx 0$.

Now, we are ready to introduce the concept of approximate invariance.

Precisely, the approximate equation

$$
F(z, \epsilon) \approx 0
$$

is said to be invariant with respect to the approximate group of transformations

$$
z^{\prime} \approx f_{0}(z, \epsilon, a)
$$

if

$$
F(f(z, \epsilon, a)) \approx 0
$$

for all $z$ satisfying $(\overline{\mathrm{C} 15})$. A criterion for obtaining the approximate symmetries of a given equation is expressed by

Theorem 2 Let us suppose that the function $F(z, \epsilon)=\left(F^{1}(z, \epsilon), \ldots, F^{n}(z, \epsilon)\right)$, $n<N$, which is jointly analytic in the variables $z$ and $\epsilon$, satisfies the condition

$$
\left.\operatorname{rank} F^{\prime}(z, 0)\right|_{F(z, 0)=0}=n,
$$


where $F^{\prime}(z, 0)=\left\|\frac{\partial F^{\nu}(z, 0)}{\partial z^{i}}\right\|$ for $\nu=1, \ldots, n$ and $i=1, \ldots, N$. For the equation

$$
F(z, 0)=o\left(\epsilon^{p}\right)
$$

to be invariant under the approximate group of transformations

$$
z^{\prime}=f(z, \epsilon, a)+o\left(\epsilon^{p}\right)
$$

with infinitesimal operator

$$
X=\xi(z, \epsilon) \frac{\partial}{\partial z}, \quad \xi=\left.\frac{\partial f}{\partial a}\right|_{a=0}+o\left(\epsilon^{p}\right)
$$

it is necessary and sufficient that

$$
X F(z, \epsilon)=o\left(\epsilon^{p}\right)
$$

whenever $F(z, \epsilon)=o\left(\epsilon^{p}\right)$.

In order to solve Eq. (C18) to within $o\left(\epsilon^{p}\right)$ one needs to represent $z, F$ and $\xi^{k}$ in the form

$$
\begin{gathered}
z \approx y_{0}+\epsilon y_{1}+\ldots+\epsilon^{p} y_{p}, \quad F(z, \epsilon) \approx \sum_{i=0}^{p} \epsilon^{i} F_{i}(z) \\
\xi^{k}(z, \epsilon) \approx \sum_{i=0}^{p} \epsilon^{i} \xi_{i}^{k}(z) .
\end{gathered}
$$

For $p=1$, we obtain

$$
\begin{gathered}
\left.\xi_{0}^{k}\left(y_{0}\right) \frac{\partial F_{0}}{\partial z^{k}}\right|_{z=y_{0}}=0 \\
\left.\xi_{1}^{k}\left(y_{0}\right) \frac{\partial F_{0}}{\partial z^{k}}\right|_{z=y_{0}}+\left.\xi_{0}^{k}\left(y_{0}\right) \frac{\partial F_{1}}{\partial z^{k}}\right|_{z=y_{0}}+\left.y_{1}^{l} \frac{\partial}{\partial z^{l}}\left[\xi_{0}^{k}(z) \frac{\partial F_{0}}{\partial z^{k}}\right]\right|_{z=y_{0}}=0
\end{gathered}
$$

under the conditions

$$
F_{0}\left(y_{0}\right)=0, \quad F_{1}\left(y_{0}\right)+\left.y_{1}^{l} \frac{\partial F_{0}(z)}{\partial z^{l}}\right|_{z=y_{0}}=0
$$




\section{References}

${ }^{1}$ C. Lebrun, J. Diff. Geom. 34, 223 (1991).

${ }^{2}$ See, for example: M.V. Saveliev, Theor. Math. Fiz. 92, 457 (1992), and references therein.

${ }^{3}$ I.M. Krichever, The $\tau$-function of the Universal Whitham Hierarchy, Matrix Models and Topological Field Theories, Preprint LPTENS-92/18, Paris 1992.

${ }^{4}$ V.A. Baikov, R.K. Gazizov and N.H. Ibragimov, Mat. Sbornik 136, No.4,435 (1988) [English trans. in Math. USSR Sbornik, vol. 64, No. 2 (1989)].

${ }^{5}$ P.J. Olver, Applications of Lie Groups to Differential Equations (Springer, New York, 1986).

${ }^{6}$ H.D. Wahlquist and F.B. Estabrook, J. Math. Phys. 16, 1 (1975).

${ }^{7}$ M. Abramowitz and I. Stegun (Eds.), Handbook of Mathematical Functions (Dover, New York, 1972).

${ }^{8}$ B.G. Wybourne, Classical Groups for Physicists (J. Wiley and Sons, N.Y, 1974).

${ }^{9}$ W. Miller jr., Lie Theory and Special Functions (Academic, N.Y. 1968).

${ }^{10}$ B.K. Harrison and F.B. Estabrook, J. Math. Phys. 12, 653 (1971).

${ }^{11}$ G.W. Gibbons and S.W. Hawking, Phys. Lett. B 78, 430 (1978). 